\title{
Design and Performance Evaluation of Modular Compliant Mechanisms
}

\author{
Santosh B. Jadhav*, Kishor K. Dhande, Suhas P. Deshmukh \\ Rajarshi Shahu College of Engineering, Pune, India \\ * Corresponding author email: hisantosh2009@gmail.com \\ Received: 02 August 2018 / Revised: 05 November 2018 / Accepted: 15 November 2018 / Published: 16 November 2018
}

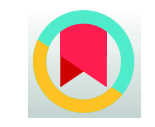

\begin{abstract}
In this study, modular design for compliant stage using a parallelogram flexure module is proposed. The material of the proposed stages chosen is Aluminium alloy T6-6051 due to excellent phase stability, low internal stresses, low density etc. The stage is driven by a linear electromagnetic actuator i.e. voice coil actuator. The preliminary static structural analysis is used to determine the range of motion, stiffness by considering stress limitations. FEA results show a maximum range of motion up to $30 \mathrm{~mm}$ without exceeding stress limit of $275 \mathrm{Mpa}$. The proposed mechanisms provide low stiffness in the degree of freedom directions and provide large stiffness in the degree of constraint direction.so it is adequately constrained. The stage provides high precision and repeatability with an increased range of motion, minimal parasitic motions (i.e.150 microns) and limited cross axes coupling.
\end{abstract}

Keywords: Compliant, range of motion, buckling, parasitic motions, cross axes coupling etc

\section{Introduction}

Flexure mechanisms are used totransmit motion through elastic deformation of flexure unit instead of conventional rigid joints [1]. In this built mechanism wasted tested with weight pan method and the results were in close match with analytical and experimental [2]. The monolithic structure eliminates completely friction, wear and backlash which provides highly repeatable motion. The benefits include design simplicity, without maintenance and improved life, has carried out the parametric analysis of the model in XY stage [3].Due to these Benefits, flexure mechanisms are used in several applications such as multi-axis flexure mechanisms used in precision alignment, insensors and actuators, energy harvesting devices, micro/nano-manipulators, scanningprobe systems for precision metrology and nanomanufacturing.Flexure mechanism is obtained using serial and parallel kinematic configuration. By simply stacking one single-axis system on top of another, serial kinematic configuration is obtained to achieve the desired DOF. These configurations are bulky, complexdue to moving cables and actuators which badly affect dynamic performance. On the other hand, Parallel kinematic configuration uses ground-mounted actuators which are compact and simple in construction. The main drawbacks as compared to serial kinematic designs are smaller motion range, possibilityof over-constraint, and large error motions [4]. Nanopositioning systems are used to provide nanometric precision, accuracy and resolution in scanning probe based microscopy, manipulation and manufacturing. Due to lack of friction and backlash, flexure mechanisms are the most common bearing choice for nanopositioning systems [5]. In this the overview of the flexure mechanism by different researchers was been done [6]. However, most existing flexurebased multi-axis nanopositioning systems are capable of approximately a $100 \mu \mathrm{m}$ range of motion per axis. The challenge is not only create a multi-axis flexure mechanism which able to provide large motion range, but also in the mechanical integration of the flexure mechanism with ground-mounted actuators.Since flexure mechanisms provide the motion guidance attributes of mechanisms with the elastic elements of structures [7]. In this optimization was been carried out on the motion stage in micro scale [8]. Synthesis is based on a systematic and symmetric layout of constraints that are realized by means of common flexure building blocks. 
Design and Performance Evaluation of Modular Compliant Mechanisms

An analytical formulation incorporating geometric nonlinearities is used in deriving the characteristics of these flexure building blocks. The prototype flexure stage has a $5 \mathrm{~mm}$ x $5 \mathrm{~mm}$ range of motion, with cross-axis errors of the order of one part in one thousand, and motion stage yaw errors of the order of a few arc seconds [9-10]. Presents parallel kinematic XY flexure mechanism designs based on systematic constraint patterns that allow large ranges of motion $(5 \mathrm{~mm} \times 5 \mathrm{~mm})$ without causing over-constraint or significant error motions. Comparisons between closed form linear and non-linear analyses are presented. It is shown that geometric symmetry in the constraint arrangement relaxes some of the design tradeoffs, resulting in improved performance [11]. The non-linear analytical predictions are validated by means of computational FEA and experimental measurements [12]. Novel bearing based on a parallel-kinematic XY flexure mechanism.Inparticular, dynamic nonlinearities associated with the large displacements of the flexure mechanism and resulting controls challenges are identified. The physical system is fabricated, assembled and tested to validate its simultaneous large range and nano-metric motion capabilities. Also presented family of $\mathrm{XY}$ flexure mechanisms with large ranges of motion, first-order decoupled degrees of freedom, and small parasitic error motions. In this article the experimental work is carried out on the flexure mechanism and the results are compared in $\mathrm{X}$ and $\mathrm{Y}$ direction with FEA analysis, finds the close match with each other. The flexure mechanism is used for the precision applications. Geometric decoupling ensures large motion range along each translational direction and enables integration with large-stroke ground-mounted linear actuators. Proposed the monolithic decoupled XYZ compliant parallel mechanisms for multifunction applications, which can be fabricated monolithically without assembly and has the capability of kinetostatic decoupling. It is based on a decoupled 3-PPPR parallel kinematic mechanism. Three types of applications: motion/positioning stages, force/acceleration sensors and energy harvesting devices. Qingsong Xu, Yangmin Li [13] has carried out performance evaluations of XYZ compliant parallel manipulators in terms of statics and dynamics which is necessary in designing the CPM considering the stiffness and natural frequency requirements. Here dSPACE was used and simulink model was built and force deflection was plotted [14]. The present work focused on the systematic conceptual design of large-range translational CPMs with distributed-compliance. Compliant parallel modules with distributed-compliance, such as beam flexure, parallelogram module and double parallelogram module etc. Identified as building blocks of translational CPMs.A new parallel kinematic flexure mechanism which provides perfectly decoupled motions along the three translational directions $\mathrm{X}, \mathrm{Y}$ and $\mathrm{Z}$ and provides high stiffness along the three rotational directions $\theta_{x}, \theta_{y}$ and $\theta_{z}$ is presented. Large motion range along each translational direction is ensured and can integrate with linear actuators having large stroke depending on the need. The conceptual design which is based on a systematic arrangement of multiple rigid stages and parallelogram flexure modules is analyzed via finite elements analysis (FEA). The analysis demonstrates an XYZ motion range of $15 \mathrm{~mm} \times 15 \mathrm{~mm} \times 15 \mathrm{~mm}$. The proposed CMs includes modulardesign for 2-DOF compliant parallel manipulator, improved modular XYZ CPMs using identical parallelogram beam modules. The proposed concept is resulting in large translational motions along the $\mathrm{X}, \mathrm{Y}$, and $\mathrm{Z}$ directions, and exhibits small error motions The parameters of Length stiffness width are compared. FEA analysis is carried out on the $Z$ section model. G.Hao [15]. S Sollapur [16] in this article the static and dynamic analysis is carried out. The state space model is built and theoretical modeling is done using assumed mode methods. FEA analysis is carried is carried out on Flexure mechanism.

\section{Conceptual Design}

All the design configurations of the mechanisms utilize the same compliant flexure module i.e. parallelogram flexure module. The size of the flexure strip in all mechanisms is maintained same.Fig.1 shows three directional motion mechanisms.it is based on 3 PPP kinematic configuration using three prismatic joints.Fig.2 and Fig.3 shows planar motion mechanism based on 2 PP kinematic configuration using 2 prismatic joints. 


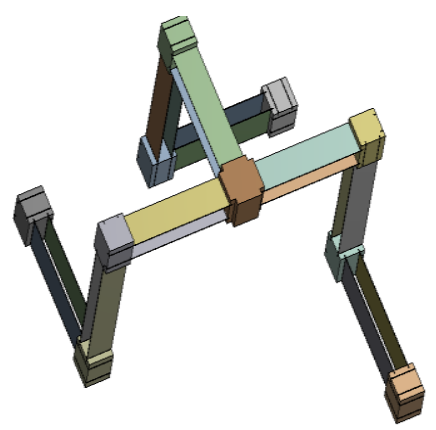

Figure 1:XYZ Mechanism

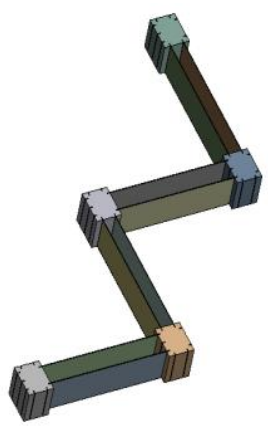

Figure 2: $X Y$ Mechanism design 1

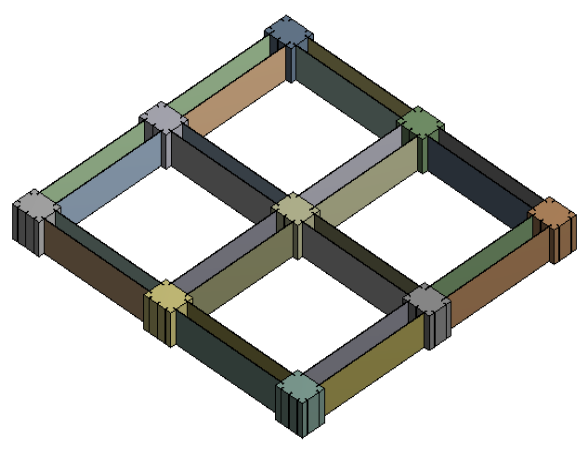

Figure 3:XY Mechanism design 2

\section{Finite Element Analysis}

Preliminary static analysis in Ansys workbench environment is carried out to evaluate the motionrange without exceeding permissiblestress limits in the flexure. The overall size of XY stage is $500 \mathrm{X} 500 \mathrm{~mm}$. Material is Aluminium alloy AL 6061-T6 chosen due to its low internal stresses, high Young's modulus to Yield stress ratio and good phase stability.

\subsection{Static Analysis of Three Direction Motion (XYZ)Stage}

Fig.4 shows CAD model is generated in Catia V20. Fig.5 shows the auto generated meshing in ansysworkbench andFig. 6 shows the boundary conditions of XYZ stage in which end three blocks are fixed and force of 100 $\mathrm{N}$ is applied in y direction.

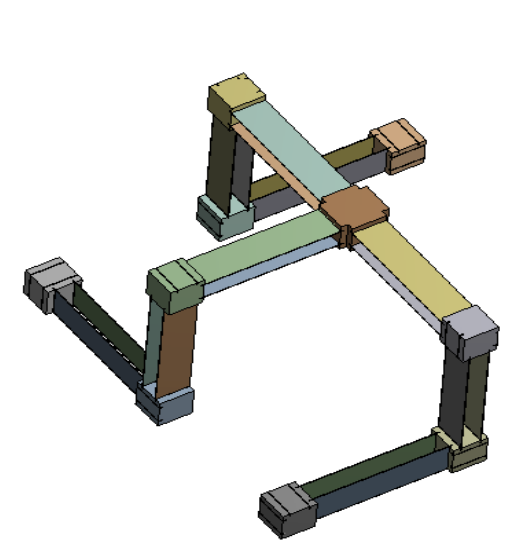

Figure 4: $C A D$ model

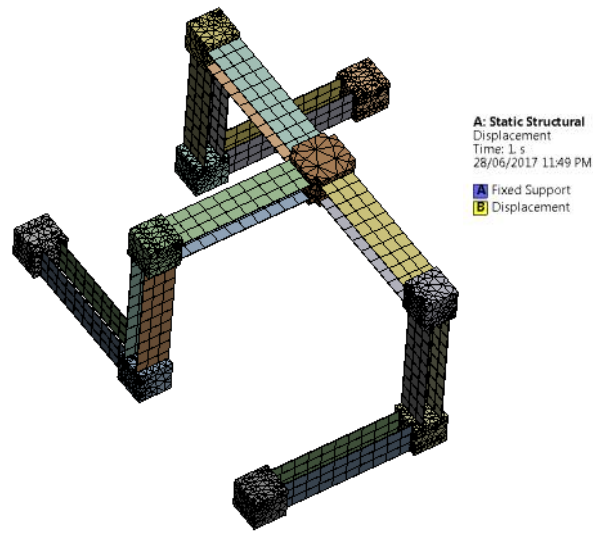

Figure 5: Meshed model

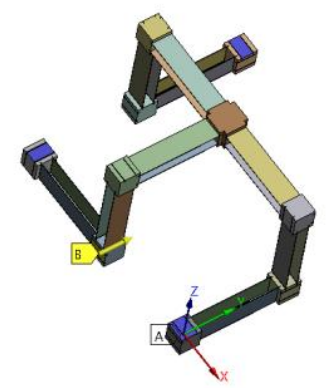

Figure 6: Boundary conditions
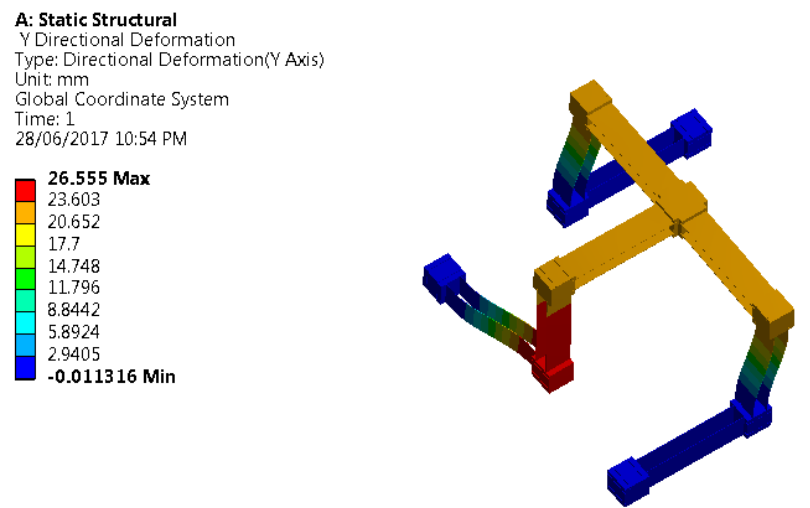

Figure 8: $Y$ directional deformation for $F Y=100 \mathrm{~N}$

Figure 7: $X$ directional deformation for $F Y=100 \mathrm{~N}$ 

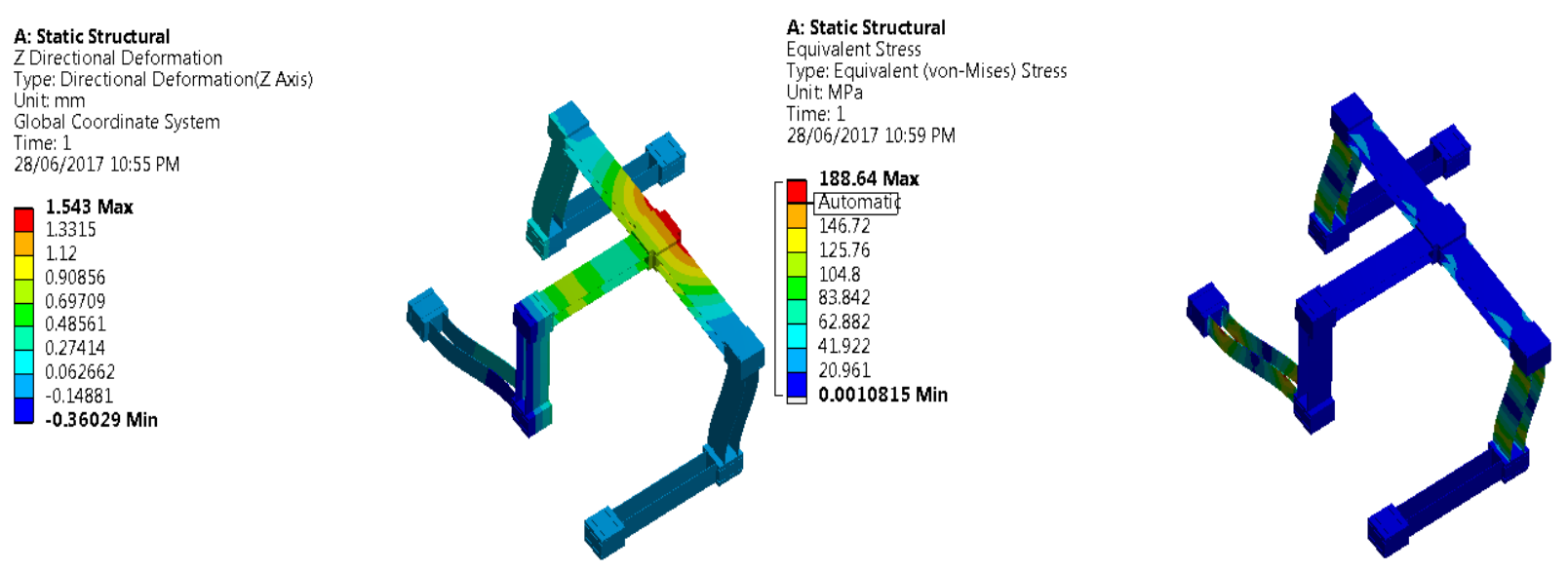

Figure 9: $Z$ directional deformation for $F Y=100 \mathrm{~N}$

Figure 10: Stress for $F Y=100 \mathrm{~N}$

Fig.7 showsdeformation in $\mathrm{X}$ direction with input force of $100 \mathrm{~N}$ in $\mathrm{Y}$ direction which gives error motion in $\mathrm{x}$ ditrections; Fig. 8 shows deformation in Y direction with input force of $100 \mathrm{~N}$ in Y direction which gives desired motion in Y directionAlso Fig.9 shows the deformation in Zdirection respectively for Force of $100 \mathrm{~N}$ in $\mathrm{Y}$ direction. From the above results, stiffness in DOF and DOC directions are found. Fig.10 shows stress developed in the mechanism under force of $100 \mathrm{~N}$ in $\mathrm{Y}$ direction. The stress value is $188 \mathrm{Mpa}$ which is much lower than the permissible stress i.e $275 \mathrm{Mpa}$ for the given material.

\subsection{Static Analysis of XY Mechanism Design 2}

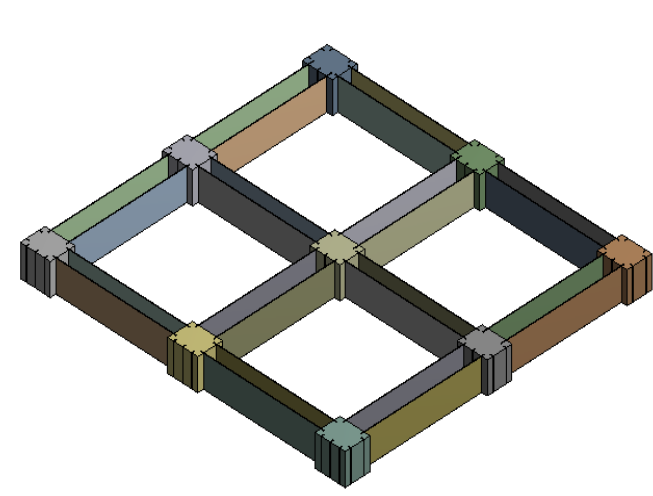

Figure 11: $C A D$ model,
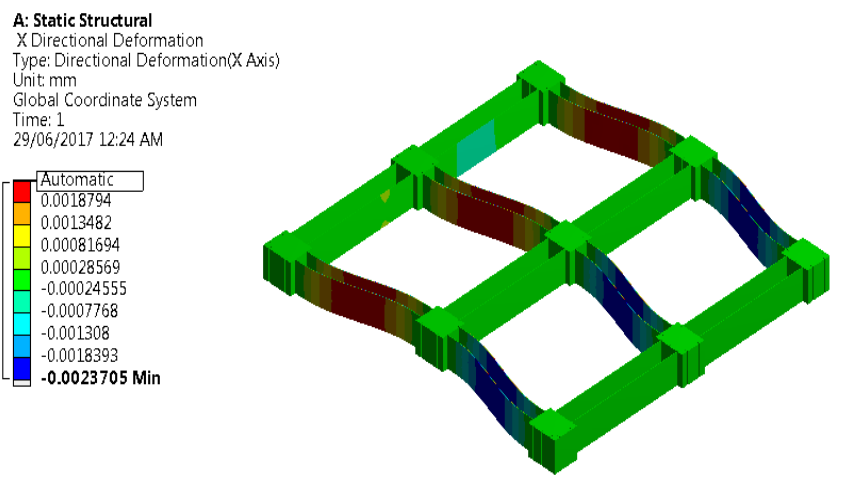
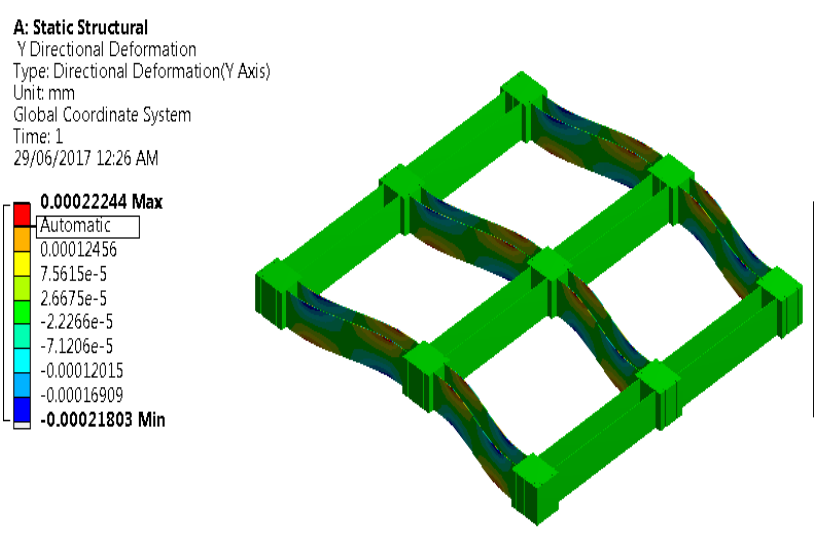

Figure 13: $Y$ directional deformation for $F Z=-100 \mathrm{~N}$
Figure 12: $X$ directional deformation for $F Z=-100 N$,
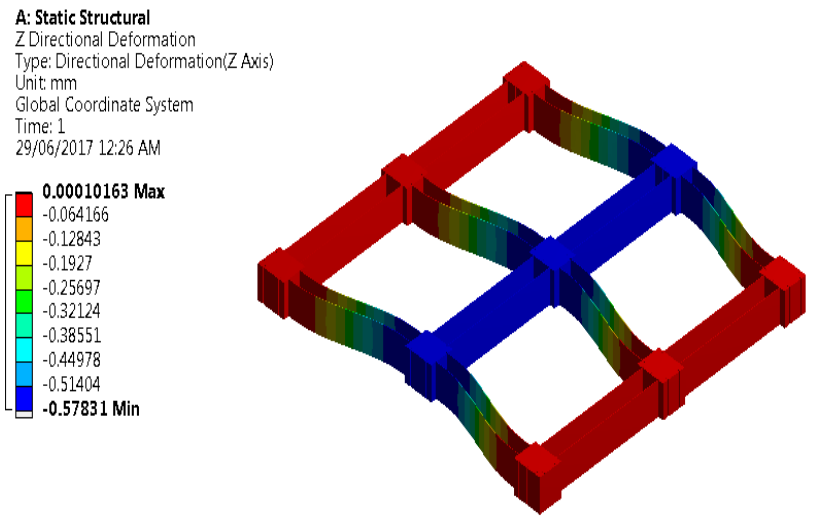

Figure 14: $Z$ directional deformation for $F Z=-100 N$, 


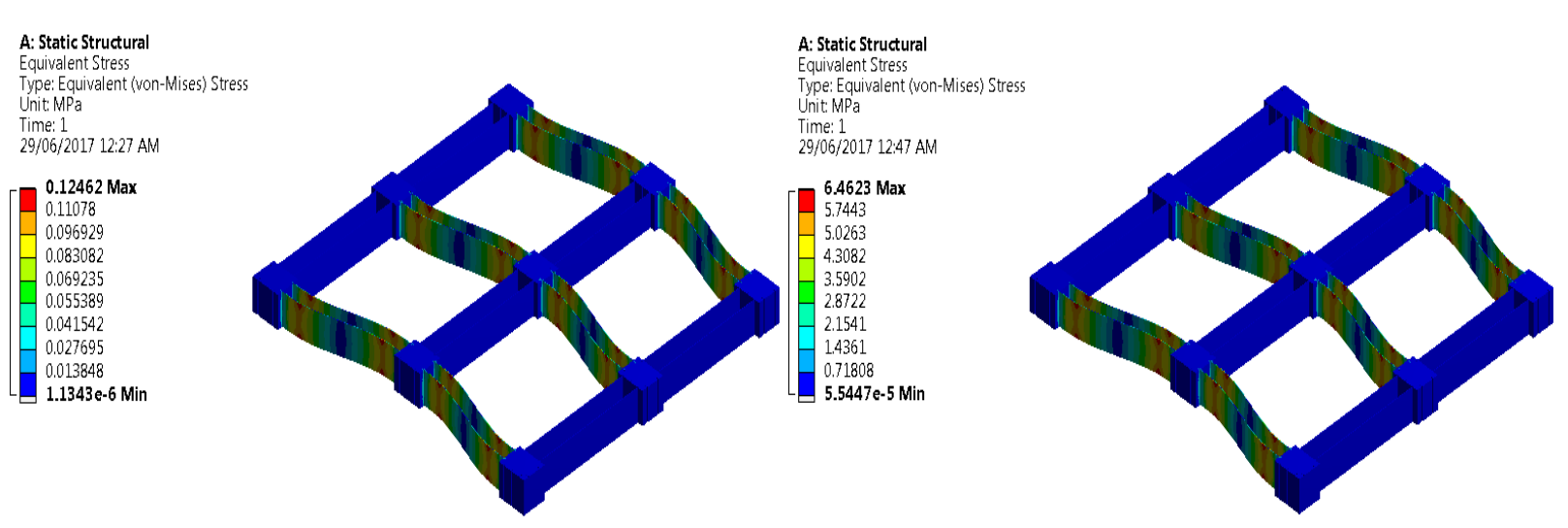

Figure 15: Stress for $F Z=-100 N$,

Figure 16: Stress for -30mm input $Z$ direction displacement

Fig. 11 shows the CAD model of XY stage generated in catia.Fig.12 shows the deformation in $\mathrm{X}$ directions for the input force of $100 \mathrm{~N}$ in $\mathrm{z}$ direction. Fig. 13 shows the deformation in Y direction for the input force of 100 $\mathrm{N}$ in $\mathrm{z}$ direction.Fig.14 shows the deformation in $\mathrm{Z}$ direction for the input force of $100 \mathrm{~N}$ in $\mathrm{z}$ direction. Fromwhich, stiffness in DOF and DOC is obtained. Fig.16 shows the stress produced for input $z$ direction displacement $30 \mathrm{~mm}$. The stress value is $6.4 \mathrm{Mpa}$ which is lower than permissible stress i.e. $275 \mathrm{Mpa}$.

\section{Conclusion}

Parallelogram flexure module is used as building block for the compliant micro motion XY stage. Novel modular design of three different configurations of planar and three directional motion stage is proposed which offers flexibility in manufacturing and ease of assembly. The Compliant XY stage delivers motion range upto $30 \mathrm{~mm}$ without exceeding the allowable stress limits. There exist limited kinematic coupling along other axes with parasitic error motions of $100 \mu \mathrm{m}$. Preliminary static structural analysis is carried out to determine the displacement range.

\section{How to Cite this Article}

S. Jadhav, K. Dhande, and S. Deshmukh, "Design and Performance Evaluation of Modular Compliant Mechanisms", J. Mod. Sim. Mater., vol. 1, no. 1, pp. 24-29, Nov. 2018. Doi: 10.21467/jmsm.1.1.24-29

\section{References}

[1] Shorya Awtar, An XYZ Parallel-Kinematic Flexure Mechanism with Geometrically Decoupled Degrees of Freedom, Journal of mechanisms and robotics, Vol.No-5, pp.10-17, 2013.

[2] Shrishail Sollapur, M S Patil, S P Deshmukh, Design and Development Aspects of Flexure Mechanism for High Precision Application, AIP Conference Proceedings 1943, 020023, 2018

[3] S. Sollapur, M. Patil, S. Deshmukh, Evaluation of Stiffness and Parametric Modeling of XY Flexure Mechanism for Precision Applications, Journal of Modeling and Simulation of Materials, vol. 1, no. 1, pp. 8-15, 2018.

[4] Xavier Hope et.al. On a simplified nonlinear analytical model for the characterization and design optimization of Compliant XY micromotion stage, Journal of Robotics and computer integrated manufacturing, pp.: 66-76, 2018.

[5] X. Zhang and Qing song Xu, Design of a New Flexure-Based XYZ Parallel Nanopositioning Stage, Proceedings of 2015 IEEE conference on robotics and biomimetic, Zuhai china, 2015.

[6] Pratik M. Waghmare, Shrishail B.Sollapur, M.S.Patil, S.P.Deshmukh, “A REVIEW PAPER ON FLEXURE”, International Journal for Science and Advance Research in Technology, Vol 3, Issue 10, 2017.

[7] 7.Dan Zhang, Zhen Gao, A novel flexure parallel micromanipulator based on multilevel displacement amplifier, SENSORDEVICES, the third international conference on sensor device, technologies, 2012.

[8] S. Lin, Y. Jia, Q. Xu, Design and optimization of a long stroke compliant micro positioning stage driven by voice coil motor, Proceeding of $12^{\text {th }}$ international conference on control automation Robotics and vision (ICARCV), pp.1716-1721,2012. 
[9] Hopkins, J.B and Culpepper, M.L, Synthesis of Multi-Degree of Freedom, Parallel Flexure System Concepts via Freedom and Constraint Topology (FACT). Part I: Principles, Precision Engineering, Vol. 34(1):259-270, 2010.

[10] Kim C. J, Moon Y. M., Kota S, A Building Block Approach to the Conceptual Synthesis of Compliant Mechanisms Utilizing Compliance and Stiffness Ellipsoids, Journal of mechanical Design, Vol. 130(2): 022308,2008.

[11] Awtar, S. Slocum, A.H., Characteristics of Beam-Based Flexure Modules, Journal of Mechanical Design, Vol. 129(6): 624-639,2007.

[12] Li Y, Xu Q, Design and Optimization of an XYZ Parallel Micromanipulator with Flexure Hinges, Journal of Intelligent and Robotic Systems, Vol. 55(4-5): 377-402, 2009.

[13] Li Y., Xu Q., A Totally Decoupled Piezo-Driven XYZ Flexure parallel micro positioning Stage for Micro/Nanomanipulation, IEEE Transactions on Automation Science and Engineering, Vol. 8(2): 265-279,2011.

[14] Shrishail B Sollapur, Dr. M S Patil and Dr. S P Deshmukh, Experimental Investigation of High Precision XY Mechanism, International Journal of Mechanical Engineering and Technology,9(5),pp.43-50,2018.

[15] G.Hao, "Towards the design of monolithic decoupled XYZ compliant parallel mechanisms, for multifunction applications", Journal of mechanical sciences,pp-291-302, 2013.

[16] Sollapur Shrishail B and Deshmukh Suhas P, "XY Scanning Mechanism: A Dynamic Approach," International Journal of Mechanical Engineering and Robotics Research, Vol.3, No.4, pp. 140-154, 2014.

\section{Publish your research article in AIJR journals- \\ $\checkmark$ Online Submission and Tracking \\ $\checkmark$ Peer-Reviewed \\ $\checkmark$ Rapid decision \\ $\checkmark$ Immediate Publication after acceptance \\ $\checkmark$ Articles freely available online \\ $\checkmark$ Retain full copyright of your article. \\ Submit your article at journals.aijr.in}

Publish your books with AIJR publisher-

$\checkmark$ Publish with ISBN and DOI.

$\checkmark$ Publish Thesis/Dissertation as a Book.

$\checkmark$ Publish Monograph.

$\checkmark$ Publish Edited Volume/ Book.

$\checkmark$ Publish Conference Proceedings

$\checkmark$ Retain full copyright of your books.

Submit your manuscript at books.aijr.org 